years and stressed that, although the methods of computation vary considerably for different tables, none of them appears to be correct. All are based on work done half a century ago in Germany by Rubner and in the United States by Atwater, who had been a pupil of Rubner. Both these early pioneers measured the intakes of protein, fat, carbohydrate and calories of people on different diets and estimated the calories lost in the frees and urine. From their results they calculated the energy values of individual foods and of mixed diets. In 1899 Atwater and Bryant concluded that protein and carbohydrate each give 4.0 kcal./gm. of total nutrients, and fat $8.9 \mathrm{kcal} . / \mathrm{gm}$. ; and yet a few years later when they compiled tables of food values for the composition of several thousand foodstuffs, they used Rubner's earlier figures of $4 \cdot 1$ for protein and carbohydrate and $9 \cdot 3$ for fat-a strange inconsistency which they did not explain and which was the forerunner of many others by later compilers of food tables. Indeed, throughout the years as one error was corrected another appeared to creep in. Even as recently as 1947 the committee on calorie conversion factors and food composition tables of the United Nations Food and Agriculture Organization, in calculating the calories per gram of ingested protein, failed to apply properly a simple correction factor for the amount of unoxidized material excreted in the urine, and thus, while improving some portions of the general computation, introduced a new error in another portion.

Towards the end of her paper Dr. Widdowson dwelt on the great need for further experiments with mixed diets more typical of those eaten to-day than were the diets studied by Atwater. She also stressed the need for experiments to be done with larger numbers of people so that more information can be obtained regarding the sort of variation that normally exists between individuals in the number of calories they obtain from their food. She referred briefly to the need for evolving methods by which the physiological energy value of food in infancy and during growth can be assessed. She readily admitted that many mistakes have been made in computing the energy values of human foods; but endeavoured to console herself and those present by recalling that he who makes no mistakes rarely makes anythingcertainly not food tables.

The second paper of the afternoon session was given by Miss D. F. Hollingsworth (Ministry of Agriculture, Fisheries and Food), who carried the general problem to its next stage by discussing the difficulties which are encountered in interpreting tables of food composition and in using them to estimate the energy value of human diets. For example, before the energy values given in tables can be applied in practice it is essential to consider how much of the purchased food is edible, how much water it contains, how much is lost in cooking and serving, and, as Miss M. Andross pointed out later in the discussion, how much is left as waste on the plate. To take one example, the moisture content of flour in food tables is usually assumed to be 12 per cent whereas in Britain for other purposes it is more normally taken as 15 per cent; this leads to a difference of roughly 3 per cent in the calculated energy value of the flour. Again, the fat content of meat can vary over a very wide range, and this can have a marked effect on the energy value computed for it. With made-up dishes the problems become still more involved, and in estimating requirements at an international level further complications arise when figures, which have been computed for British food supplies, are used for estimating the requirements of other countries where higher proportions of the total energy of the diet may be supplied by carbohydrates.

The four papers and the subsequent discussion achieved what was no doubt one of the main objects of the symposium, namely, to bring out some of the numerous difficulties that arise when attempts are made to assess accurately the energy values of foods, and to indicate some of the numerous problems that still wait to be solved by chemist, biochemist, physiologist and dietitian, as well as by those whose difficult duty it is to consider the general subject of world food supplies, their economics and distribution. J. A. B. SмIтr

\section{CHEMICAL ASPECTS OF SEMICONDUCTORS}

$\mathrm{A}$

HALF-DAY symposium on "Chemical Aspects of Semiconductors" was held in the Chemistry Department of the University of Reading on March 26. The meeting, which attracted a gathering of some two hundred, was arranged by Dr. P. F. Holt (Reading) on behalf of the London Section of the Royal Institute of Chemistry. The chair was taken by Dr. F. S. Stone (Bristol). The aim of the symposium was to bring to the attention of chemists some of the problems of a chemical nature arising in the semiconductor field. The rapid developments in the technology of semiconducting materials which have taken place during the past ten years have only been achieved by close co-operation between the chemist and the physicist, and it is important that from time to time the current chemical problems in this predominantly physical field should be presented in a chemical forum. There is, moreover, a growing awareness among chemists of the contribution which semiconductors can make to fundamental studies on essentially chemical problems, such as adsorption and chemical bonding in solids, and this factor no doubt added to the general interest which the meeting aroused.

Since many of those present were not specialists in the semiconductor field, the main contributions were preceded by some introductory remarks on semiconductors, given by Dr. F. S. Stone. After outlining the principles underlying intrinsic and impurity semiconductivity, Dr. Stone dealt briefly with the rectifying and transistor action of germanium. In reviewing the chemical aspects, he stressed the importance of purification and standardization of materials. The significance of adsorption of active gases and vapours in creating a space charge at semiconductor surfaces was also emphasized, and attention was directed to the fact that very little fundamental work has so far appeared on adsorption at silicon and germanium surfaces.

The first of the more specialized papers was read by Mr. I. M. Ross (Services Electronics Research Laboratory, Baldock), who reviewed work on intermetallic semiconductors. Useful as silicon and germanium are for crystal-valve applications, their characteristics in respect of the combination of carrier mobility and separation between the valence and conduction bands are not entirely satisfactory. For other applications, such as photo-devices, quite different characteristics may be required. Interest has therefore turned to the closest analogues of 
silicon and germanium, namely, compounds between elements in Group III and Group V (for example, InSb, GaAs) crystallizing in the zinc blende structure. Like the purely covalent germanium and silicon, the bonds in these 'III/V compounds' are also mainly homopolar. Mr. Ross pointed out that the band. separation in these compounds covers a wide range of values and that electron mobilities are in general high. With InSb, for example, which has been the most widely studied of these compounds, the electron mobility is $85,000 \mathrm{~cm} .{ }^{2} /$ volt-sec. at $300^{\circ} \mathrm{K}$., compared with $3,600 \mathrm{~cm} .^{2} /$ volt-sec. for germanium. The band-separation in this case is too low for transistor action at room temperature, but the high mobility has led to a number of new applications, particularly concerned with the Hall effect, in which the available power is some three hundred times that given by germanium. Mr. Ross demonstrated this to the meeting by showing the effect of the earth's magnetic field upon the current flowing in a crystal of InSb. $\mathrm{H}_{\Theta}$ also outlined some of the technical problems associated with producing crystals of III/V compounds. Zone-refining (see below) is in general difficult to apply to these compounds due to their tendency to decompose, and, with the exception of InSb, the problems of purification and growth of single crystals have not been satisfactorily solved. In $\mathrm{GaAs}$, for example, reliance has had to be placed on extensive purification of the parent elements. A further problem, the study of which has only just begun, is that of obtaining stoichiometry in these compounds.

A contribution which aroused much interest was made by Mr. A. A. Smales (Atomic Energy Research Establishment, Harwell), who reviewed methods for estimating trace impurities. Besides sensitivity, specificity and accuracy, Mr. Smales classified the various methods according to their advantages in respect of freedom from contamination (either adventitious or from reagents) and their ability to deal with several elements simultaneously. Emission spectroscopy has this latter advantage and is highly specific. Freedom from contamination may be avoided if the material is examined directly, but not if impurities have been chemically concentrated before determination, to enhance the sensitivity. The most sensitive methods are undoubtedly the isotope dilution technique, employing mass-spectrometric analysis, although the contamination problem still exists there, and the radioactivation method, which involves neutron irradiation in a pile followed by radiochemical separation. For many elements these methods have sensitivities better than 1 part in $10^{9}$. The first technique is applicable only to those elements for which separated isotopes are available, while the second is limited to those with suitable nuclear characteristics ; nevertheless, more than half the elements can be determined by one or the other method. Both require that elements be analysed individually, though the development of gamma-ray spectrometers will ultimately enable the radioactivation technique to be more versatile in this respect. Using the vacuum-spark ion source, the mass spectrometer may be applied to the determination of several elements simultaneously, but the sensitivity is greatly reduced. Mr. Smales also described the more conventional methods, such as colorimetry and polarography, pointing out that although they are fairly sensitive $(0 \cdot 1-1$ p.p.m.), the reagent blank problem remains a major limitation.

Mr. H. J. Cluley (General Electric Co., Ltd., Wembley) described chemical processes for obtaining germanium and gilicon in high purity. Germanium is extracted as the tetrachloride from coal (flue dusts) or from zinc blende during smelting. Purification is then effected by fractional distillation and refluxing over copper or by repeated distillation. Arsenic, the main impurity, is thereby reduced in concentration to less than 1 part in 10 million. The metal is obtained by hydrolysis to germanium oxide and reduction with hydrogen. High-purity silicon may be prepared by fractionation of silicon tetraiodide, followed by the van Arkel technique of thermal decomposition at a hot filament. A second method is the reduction of silicon tetrachloride with zinc in the vapour phase. Mr. Cluley also reviewed other methods involving the thermal decomposition or hydrogen reduction of silicon compounds. Before germanium and silicon can be used for electrical work, further purification by zone-refining is necessary. The details of this technique were described by Mr. S. E. Bradshaw (General Electric Co., Ltd., Wembley). In zonerefining, a molten zone is slowly but repeatedly made to pass along an ingot of the material. The effect is to concentrate the impurity at one end since, when an impure solid is melted, the impurity concentration in the solid $\left(c_{s}\right)$ differs from that in the melt $\left(c_{L}\right)$ with which it is in equilibrium. If the segregation coefficient $K\left(K=c_{s} / c_{L}\right)$ is less than unity, the impurity is swept in the direction of traverse. If $K$ is greater than unity, the impurity concentrates in the regions where freezing begins. Unless $K$ happens to be close to unity (for example, boron in silicon), the method is extremely effective and impurity concentrations can readily be reduced to 1 part in $10^{8}$.

A paper by Mr. J. I. Carasso (Post Office Research Station, Dollis Hill) dealt with two chemical factors affecting the performance of germanium diodes. The first of these was the use of nickel as an additive for the controlled shortening of carrier life-times, a requirement for high-frequency operation. It is found that the addition of nickel to the melt during the crystal-growing stage does not produce the required degree of control, the resulting concentrations of nickel in germanium being different from those expected on the basis of a simple treatment of the segregation coefficiont. This anomaly is explained by a suggested diffusion of nickel atoms through the germanium lattice. Direct addition of nickel to germanium by lattice diffusion from a nickel/germanium interface may, in fact, be a more satisfactory method of controlling the incorporation, and Mr. Carasso described experiments of this type at present in progress at Dollis Hill. The second topic treated by Mr. Carasso was surface contamination. Adsorbed gas may exert an influence upon a $p-n$ junction due to surface mobility of charged particles. Or again, the space charge arising at the surface may itself be sufficiently intense to form, for example, a $p$-type 'skin' on n-type material (channel effect). Thirdly, opportunity is provided for high rates of carrier recombination at the surface. Mr. Carasso discussed specifically the behaviour of water vapour and iodine vapour, showing that the channel effect for water is opposite to that for iodine.

The importance of employing single crystals of a high degree of perfection for semiconductor work is frequently emphasized. This aspect of the field was covered by Dr. E. Billig (Associated Electrical Industries, Ltd., Aldermaston), who gave a descriptive account of the methods of growing single crystals. Among other points, he discussed the correlation of heat dissipation with growth-rate and mentioned 
that the usual rates, as obtained by the standard Kyropoulos method, could be considerably increased by undercooling the melt. Melts can be undercooled by as much as $20-30^{\circ} \mathrm{C}$. without risk of nucleation. Dr. Billig showed that if one (111) plane of a germanium crystal is maintained vertical, the crystal is able to grow without the need for nucleating new layers; no experimental evidence of growth from spiral dislocations has in fact been obtained in this work. Imperfections, however, do arise, due possibly to the occlusion of impurities and to mechanical stresses. Such stresses are commonly caused by uneven temperature distribution or by certain crystallographic features such as the intersection of two twin boundaries. A single twin boundary, on the other hand, apparently has no effect on the electrical properties. Dr. Billig concluded by showing a film illustrating various features in the growth of germanium and silicon monocrystals. Under certain conditions, such crystals have been obtained in the form of thin plates which display a striking degree of external symmetry. The film also showed the extremely rapid growth of dendritic crystals in the form of thin and long lamellæ, demonstrating in a convincing manner that, even in an isotropic medium of cubic symmetry, crystal growth can take place by the deposition of individual layers orientated along the close-packed (111) planes.

F. S. STONE

\section{STAR-STREAMING FROM STARS OF SPECTRAL TYPES A AND B}

A

PAPER by T. R. Tannahill on "Star-Streaming from the Stars of Spectral Type B" (Mon. Not. Roy. Astro. Soc., 114, No. 4; 1954) gives the results of his analysis, on Eddington's two-drift hypothesis, of the proper motions of 1,536 stars of spectral types $O$ and $B$ in the Boss "General Catalogue". The material for the investigation was grouped into ten regions, each region consisting of a 'square' of north galactic latitude combined with the diametrically opposite region of south galactic latitude, as shown in the first table of the paper. An examination of this table and also of the drift-curves establishes that it is impossible to represent the motions of the $B$-type stars by a single drift, and a large proportion of the stars has been assigned to a second drift in order to make a satisfactory fit; in four out of the ten regions in Drift ii the number of stars exceeds that in Drift i. From an analysis of the data, the drift constants are derived with the probable errors.

In a previous paper (Mon. Not. Roy. Astro. Soc., 112 , No. $3 ; 1952$ ), Tannahill gave the corresponding results from the galactic zone of stars of types $A_{5}-M$, and a comparison with the present results indicates satisfactory accordance, considering the probable errors involved. The values of $L, B$ and $h W$, the galactic co-ordinates of each drift apex and the space velocities of each drift, are used to calculate the theoretical values of $h V_{c}$ and $\theta_{c}$-that is, the projections of the space-velocities of the drifts on the tangent-plane at the centre of the region, and the position angles of the directions towards the drift apices - and these are listed together with the residuals $h V_{c}-h V$ and $\theta_{c}-\theta$. Considering that only 1,536 stars were used in the investigation, the residuals are quite satisfactory except in Drift $i$, region 5 , where $\theta_{c}-\theta_{1}$ exceeds $49^{\circ}$. The linear components of the motion of Drift $i$ relative to Drift ii are given in a table, and also the galactic longitude and latitude of the vertex of star-streaming and the relative spacevelocity of the drifts. One curious feature is the close agreement of the galactic co-ordinates of the vertex position $\left(343 \cdot 3^{\circ},-1 \cdot 6^{\circ}\right)$ obtained by the investigation, and that normally found $\left(343^{\circ},-2^{\circ}\right)$. It is admitted that the closeness of the agreement must be regarded as fortuitous, on account of the probable errors involved. Nevertheless, this does not vitiate the final conclusion, and the result "removes any shadow of doubt that the $B$-type stars exhibit the phenomenon of star-streaming".

The subject is continued in a further paper (Mon. Not. Roy. Astro. Soc., 114, No. 5; 1954), "StarStreaming from the Proper Motions of the $A$-Type Stars of the Boss 'General Catalogue'", in which are given the results of the analysis of the motions of 8,676 stars of the spectral types $B_{8}-A_{3}$. The method of dealing with the data follows closely that described in the 1952 paper for stars of types $A_{5}-M$, and stars belonging to known clusters have been rejected; of these, twenty belong to the Pleiades group, forty-six to the Ursa Major group, and nine to the Taurid cluster. The same difficulty in analysis arose as with the $A_{5}-M$ stars, the regions around Drift i apex offering certain problems in analysis, especially in the determination of the Drift ii constants. The higher concentration of the stars in the galactic plane, the regions in the higher galactic latitudes being sparsely populated, presented further difficulties; but in spite of these, analysis was possible in all regions, and the results are given in nine tables.

One rather surprising feature may be mentioned: in the $A_{5}-M$ types there was a marked decrease of $h W$ with advancing spectral type, which was not maintained in the $A$-stars. There was a decided systematic change in the Drift ii apex, the right ascension decreasing, and the declination increasing, with advancing type. In addition, large and apparently irregular variations occurred in some of the quantities determined, and, judging by the probable errors involved, must have a statistical significance, yet they indicated no systematic trend. Some possible reasons for these discrepancies are suggested by Tannahill, and though they are only tentative, it is probable that future research will modify some of the hypotheses on which present investigations are based. The galactic co-ordinates of the vertex were found to be $\left(365.5^{\circ},-0.5^{\circ}\right)$, and the equatorial co-ordinates of the solar apex $\left(264 \cdot 8^{\circ},+31.5^{\circ}\right)$; the latter is not far from the co-ordinates of the solar apex as found from the streaming of the spectral type $B$ stars $\left(260 \cdot 7^{\circ}+37 \cdot 3^{\circ}\right)$.

\section{THE INSTITUTE OF SEAWEED RESEARCH}

$\mathrm{N}$ the annual report for 1954 of the Institute of Seaweed Research, the interested layman's attention will be attracted by the statement that "mechanical harvesting of sublittoral Laminariaceae has now been proved to be a feasible and reasonably cheap operation". At present, six seaweed-processing factories in Scotland use more than 30,000 tons of cast weed annually, and it is estimated that one motor fishing vessel adapted to operate twin I.S.R. belt harvesters should be able to collect more than five thousand tons of Laminaria cloustoni a year in good areas. One of the main objectives of the Institute 